\title{
THEMATIC PAPER \\ Perinatal mental health around the world: priorities for research and service development in China
}

\author{
Simone Eliane Schwank, ${ }^{1}$ Q Qiongjie Zhou, ${ }^{2}$ Yanling $\mathrm{He}^{3}$ and \\ Ganesh Acharya ${ }^{4}$
}

\begin{abstract}
'PhD, MS, MA, Msc, Clinical psychologist, Department of Clinical Science, Intervention and Technology (CLINTEC), Karolinska Institutet Stockholm, Karolinska

Sweden.

mail: simone.schwank@ki.se

${ }^{2} \mathrm{MD}$, Specialist Obstetrician, Obstetrics and Gynaecology dan University, Shanghai, China

${ }^{3} \mathrm{MD}$, MintMH, Director, Department of Psychiatric Epidemiology, Shanghai Mental Health Centre, Shanghai Jia Tong University School of Medicine, China

${ }^{4} \mathrm{MD}, \mathrm{PhD}, \mathrm{FRCOG}$, Professor and Head, Division of Obstetrics and Gynaecology, Department of Clinical Science, Intervention and Technology (CLINTEC), Karolinska Institutet, Stockholm, Sweden
\end{abstract}

Conflicts of interest: None.

Keywords. Perinatal psychiatry; anxiety disorders; community mental health teams; psychiatric nursing; stigma and discrimination.

First received 19 Jul 2019 Final revision 19 Dec 2019 Accepted 20 Jan 2020

doi:10.1192/bji.2020.5

(c) The Authors 2020. This is an Open Access article, distributed under the terms of the Creative Commons Attribution licence (http://creativecommons org/ (http://reativecom, which perg/ permits unrestricted re-use, distribution, and reproduction in any medium provided the original work is properly cited.

\begin{abstract}
China's healthcare is improving together with rapid economic growth. Yet, mental healthcare is lagging behind. Prevalence of perinatal depression is high among women of the one-child generation, but access to qualified care is limited. Chinese healthcare professionals, policy makers and patients alike express concerns about insufficient knowledge among the public as well as healthcare providers regarding mental disorders. There appears to be a general lack of help-seeking behaviour for mental disorders owing to perceived risk of social stigmatisation. Social support through family and friends, use of online resources and community healthcare services are preferred, rather than seeking help from mental health specialists.
\end{abstract}

It has been estimated that 39.6 million healthy life-years will be lost in China by 2025 because of mental illnesses (the estimate is based on disability adjusted life-years, or DALYs). ${ }^{1}$ The prevalence of mental disorders reported by the recent Chinese Mental Health Survey was higher than that reported in the previous two large-scale surveys from China and there are substantial gaps in the accessibility of care. ${ }^{2}$ The number of epidemiological studies on mental health in China is relatively small. Basic data on many mental disorders, populations and regions are still missing. There are so many knowledge gaps in this field that an attempt to fill even one or two of these gaps is valuable. The prevalence of perinatal depression in women with obstetric complications in China is estimated to be $10-15 \%^{2}$ and it is reported to be higher in women of the Chinese one-child generation compared with previous generations, particularly among women who give birth to a daughter. ${ }^{3}$ Perinatal depression appears to be more common in rural than in urban areas. ${ }^{4}$ Prevalence rates are highest in the rural areas' prefecture-level cities (25.4\%), lower in the provincial capitals $(19.5 \%)$ and lowest in the municipalities directly under central government $(12.9 \%) .^{5}$ However, epidemiological research shows that women in metropolitan areas are especially at risk for developing common mental disorders. ${ }^{6}$

\section{Perinatal mental health in China}

Perinatal mental disorders, including suicide, are emerging as a major cause of maternal mortality in both high- and low-income countries. In China, however, community members rarely consider mental illness a direct cause of suicide - it is typically seen as the result of family conflict or other social stressors. Exact national statistics on perinatal mental disorders are not available for China, but mental illness-related maternal mortality is reported to be negligible in Shanghai. ${ }^{7}$ The results of a recently published retrospective study on postpartum depression among Chinese women are consistent with previous findings that the majority (84\%) of cases of postpartum depression occur within 6 weeks after childbirth and up to $94 \%$ within the first 4 months postpartum. ${ }^{8}$ This indicates higher risk for developing postpartum depression soon after childbirth, which is similar to Western countries, despite the tradition of familial support and rest after confinement, 'doing the month' (zuo yue zi). 'Doing the month' is familybased care during the period immediately after delivery, with the purpose of helping the mother to go through the physical and psychological adjustments. Its main components include avoiding cold, adequate rest, sufficient cleanliness, a balanced diet, pelvic recovery and nutrient supplements, believed to restore maternal postpartum health. ${ }^{9}$ In China, 'doing the month' has been considered to have both a positive and negative impact on maternal well-being. ${ }^{10}$

\section{Risk factors for perinatal depression in China}

Women who gave birth to female infants, in the light of China's one-child policy, were at greater risk for postpartum depression. ${ }^{11}$ This is in contrast to studies conducted in Western societies, which did not find an association between fetal gender and postpartum psychology. ${ }^{1}$ China's gender preference has become a subject of substantial discussion since the implementation of the one-child policy in 1974. The one-child policy has been suggested to lead to stronger preference for males, and disappointment over the infant's gender is a significant risk factor for perinatal depression in China. ${ }^{11}$ Whether the recently introduced two-child birth control policy will have a positive effect on women's perinatal mental health remains to be seen. 
Women experiencing postpartum depression report conflicts in attempting to conform to the Chinese traditional ideals perceived as necessary to maintain family harmony, while desiring to assert modern values such as individuality and independence. Living with extended family after childbirth is commonplace. It involves potential for relationship conflicts, as living with parentsin-law after childbirth has been described as a risk factor for perinatal depression among Chinese women. ${ }^{10}$ Higher prevalence of relationship conflicts was found in Chinese women with perinatal depression. ${ }^{10}$ Paternal perceptions of partner support and social integration reduce the risk for perinatal depression and are especially important in China, with its specific traditional extended-family dynamics. It has been reported that good postnatal care and 'doing the month' are protective factors against perinatal depression. Specific components of confinement practices might reduce psychological distress in Chinese mothers during the postpartum period, although the available evidence is inconsistent. ${ }^{10}$

\section{China's maternity healthcare system and perinatal mental health}

China currently lacks national guidelines on perinatal mental healthcare and there is no systematic referral system for perinatal mental disorders, ${ }^{12}$ although recommendations have been made to improve mental health in the 2017-2020 national guidelines issued by the Chinese Ministry of Health and the 2030 Agenda for Sustainable Development. Shanghai is an exception, with perinatal guidelines issued by the Shanghai Centre for Women's and Children's Health in 2017 focusing on maternal mental health and recommendations for perinatal screening for mental health disorders. ${ }^{12}$ However, implementation of screening and treatment are yet to be established. Shanghai Centre for Women's and Children's Health promotes perinatal mental health information through a mobile phone app targeted at women with perinatal depression. Women visiting the antenatal clinic are screened for depression and anxiety. In Shanghai currently one second-tier maternity hospital and Fudan University Obstetrics and Gynaecology Hospital, which is a tertiary hospital, have routine antenatal screening for mental health problems, which was initiated in 2018. There is, however, a rising number of community health centres expanding their perinatal mental health services. In the screening programme, women with high scores on the 9-item Patient Health Questionnaire (PHQ-9) and/or the 7-item General Anxiety Disorder scale (GAD-7) are referred to multidisciplinary teams of clinical psychologists, psychiatrists, obstetricians and midwives. During the postpartum period women with moderate depression (Edinburgh Postnatal Depression Scale, EPDS, score $>10$ ) receive online services, including access to chat groups, information (videos, reading material) on mental health, breastfeeding and the mother-infant relationship, and online therapy. Women with major depressive disorder (EPDS score $>15$ ) or other severe mental disorders are referred to the Shanghai Mental Health Centre or secondary psychiatric hospitals. Similar services are also provided in several other institutions in China in different provinces.

\section{Mental health resources and service delivery}

The World Health Organization's Mental Health Gap Action Programme (mhGAP) recommends psychological treatment as the first-line management for common non-psychotic mental disorders. $^{13}$ However, resources and access to qualified mental healthcare services are limited in China, especially in rural areas. ${ }^{14}$ The majority of mental health professionals in China work in specialty psychiatric hospitals and the number of trained mental health professionals is inadequate to meet the patients' needs and fill the treatment gap. Therefore, developing skilled human resources and enhancing the capacity of primary care services needs to be prioritised for delivering efficient mental healthcare at the community level.

\section{Traditions and societal pressures}

In Chinese social settings, postpartum care is influenced by both traditional beliefs and contemporary healthcare practices. 'Doing the month' after childbirth involves a series of practices related to the maternal role, physical activity and a traditional diet, believed to restore maternal postpartum physical and mental health. ${ }^{9}$ Parents and parents-in-law encourage new mothers to adhere to traditional practices. ${ }^{9}$ Traditionally, mothers-in-law exercised significant power in Chinese households and had a major influence on the postpartum care of their daughtersin-law. ${ }^{10}$ In urban China there is an emerging trend of hiring a 'maternity matron' (specialised nurse) at home or 'doing the month' in a special maternity care centre. These services are expensive, but may reduce the women's burden of new motherhood. Parents and parents-in-law often share some of the cost. Family members and professionals provide compassionate care and boost the confidence of new mothers through their experience and skills, which has been generally perceived as a protective factor. However, with rapid cultural, economic and educational development in China, some urban and suburban women are now beginning to question such practices and to modify traditional postpartum care. ${ }^{9}$

\section{Help-seeking behaviour}

A recent survey of Shanghai women found that over $70 \%$ did not seek help for perinatal mental health problems. ${ }^{15} \mathrm{~A}$ similar pattern is seen in the general population, and low help-seeking behaviour leading to lack of timely diagnosis and appropriate management of mental 
disorders may be associated with suicide. ${ }^{16,17}$ A majority $(80 \%)$ of the Shanghai women participating in the survey would prefer information and counselling provided via online resources such as WeChat. ${ }^{15}$ Limited availability of affordable mental healthcare services, inadequate numbers of qualified and competent mental healthcare professionals, and social stigma associated with mental illness make women prefer online resources. Capacity-building through education and training of community physicians and other healthcare professionals as well as society as a whole is required to overcome the stigma and challenges associated with caring for women with perinatal mental disorders.

\section{Research priorities}

Public health research is needed, including new epidemiological studies on perinatal depression in rural and urban China. Cost-effectiveness of treatments, including eHealth support, for common mental disorders during the perinatal period should be evaluated by properly designed studies. Studies focusing on women from the onechild generation are important because of the specific challenges associated with lack of siblings to rely on for social support and the known higher risk for developing mental disorders in this population. Research into the social support function of fathers and husbands is equally important in this regard. Further research regarding women's care-seeking behaviour for common mental disorders during the perinatal period is necessary to allocate resources in line with women's relevant needs.

\section{Conclusions}

There seems to be an urgent need for improving mental health services in China, with a focus on perinatal mental healthcare. Epidemiological, clinical as well as health services implementation research on perinatal mental health need to be prioritised. Basic mental healthcare services should be available at community health centres to make them easily accessible and affordable. Educating and training more mental healthcare professionals, particularly those engaged in primary healthcare, retaining them in the healthcare system, and continuously improving their competencies and skills are essential to meet the mental healthcare needs of pregnant women and their families in China. Considering the very high demand and clear preference for online mental health services among the younger generation of pregnant women and new mothers, it would be appropriate to allocate resources to support the expansion of eHealth services and their integration into primary care in order to bridge the gap between the high prevalence of perinatal mental disorders and limited accessibility to mental healthcare services, especially for common mental disorders. Strengthening familial and social support systems could also have a synergistic positive effect on perinatal mental health.

\section{Funding}

This work was supported by the Chinese Psychoanalytic Alliance (to S.E.S.), Swedish Association for Medical Research (S.E.S.) Swedish Medical Association (S.E.S.) and Swiss National Science Foundation (S.E.S.).

\section{Acknowledgements}

We thank Professor Ding Yan, Fudan University Obstetrics and Gynaecology Hospital, the Shanghai Centre for Women's and Children's Health, Professor Liping Zhou and Assistant Professor $\mathrm{Du} \mathrm{Li}$ for their contributions regarding the Chinese maternal healthcare system and new pathways of care.

\section{Author contributions}

S.E.S., Q.Z., Y.H. and G.A. co-wrote the manuscript.

\section{References}

1 Charlson, F. J., Baxter, A. J., Cheng, H. G., et al (2016) The burden of mental, neurological, and substance use disorders in China and India: a systematic analysis of community representative epidemiological studies. Lancet, 388, 376-389.

2 Zhao, Y., Kane, I., Mao, L., et al (2016) The prevalence of antenatal depression and its related factors in Chinese pregnant women who present with obstetrical complications. Archives of Psychiatric Nursing, 30, 316-321.

3 Xiong, R., Deng, A., Wan, B., et al (2018) Prevalence and factors associated with postpartum depression in women from single-child families. International Journal of Gynaecology and Obstetrics, 141, 194-199.

4 Wong, J. \& Fisher, J. (2009) The role of traditional confinement practices in determining postpartum depression in women in Chinese cultures: a systematic review of the English language evidence. Journal of Affective Disorders, 116, 161-169.

5 Mu, T.-Y., Li, Y.-H., Pan, H.-F., et al (2019) Postpartum depressive mood (PDM) among Chinese women: a meta-analysis. Archives of Women's Mental Health, 22, 279-287.

6 Galea, S., Uddin, M. \& Koenen, K. (2011) The urban environment and mental disorders: epigenetic links. Epigenetics, 6, 400-404

7 Gu, C., Zhang, Z. \& Ding, Y. (2011) Chinese midwives' experience of providing continuity of care to labouring women. Midwifery, 27, 243-249.

8 Yang, F., Gardner, C. O., Bigdeli, T., et al (2015) Clinical features of and risk factors for major depression with history of postpartum episodes in Han Chinese women: a retrospective study. Journal of Affective Disorders, 183, 339-346.

9 Liu, Y. Q., Maloni, J. A. \& Petrini, M. A. (2014) Effect of postpartum practices of doing the month on Chinese women's physical and psychological health. Biological Research for Nursing, 16, 55-63.

10 Wong, J. \& Fisher, J. (2009) The role of traditional confinement practices in determining postpartum depression in women in Chinese cultures: a systematic review of the English language evidence. Journal of Affective Disorders, 116, 161-169.

11 Xie, R. H., Liao, S., Xie, H., et al (2011) Infant sex, family support and postpartum depression in a Chinese cohort. Journal of Epidemiology and Community Health, 65, 722-726.

12 Baidu (2017) Shanghai Maternal Health Work Specification [in Chinese]. Baidu (https://wenku.baidu.com/view/ 77d1db3b783e0912a3162a52.html). Accessed 22 June 2019.

13 Shao, Y., Wang, J. \& Xie, B. (2015) The first mental health law of China. Asian Journal of Psychiatry, 13, 72-74. 
14 Liu, J., Ma, H., He, Y. L., et al (2011) Mental health system in China: history, recent service reform and future challenges. World Psychiatry, 10, 210-216.

15 Schwank, S. E., Gu, C., Cao, Z., et al (2018) China's child policy shift and its impact on Shanghai and Hangzhou women's decision-making. International Journal of Womens Health, 10 639-648.
Phillips, M. R., Yang, G., Zhang, Y., et al (2002) Risk factors for suicide in China: a national case-control psychological autopsy study. Lancet, 360, 1728-1736.

17 Shen, Y.-C., Zhang, M.-Y., Huang, Y.-Q., et al (2006) Twelve-month prevalence, severity, and unmet need for treatment of mental disorders in metropolitan China. Psychological Medicine, 36, 257-267.

\title{
THEMATIC \\ Perinatal mental health around the PAPER world: priorities for research and service development in France
}

\author{
Anne-Laure Sutter-Dallay, ${ }^{1} \odot$ Nine M.-C. Glangeaud-Freudenthal ${ }^{2}$ and \\ Florence Gressier ${ }^{3}$
}

${ }^{1}$ Perinatal Psychiatry Network, Adult Psychiatry Department, Charles Perrens University Hospital, and Bordeaux Population Health Ce ealth Center, INSERM 1219, Bordeaux University, France. Email: alsutter@ch-perrens.fr ${ }^{2}$ CNRS-INSERM, Paris, France ${ }^{3}$ CESP-INSERM UMR1178, Department of Psychiatry, Bicêtre , Le Kremlin Bicêtre, France

Keywords. Perinatal psychiatry; France; joint care organisation.

First received 6 Nov 2019 Accepted 3 Jan 2020

\section{doi:10.1192/bji.2020.18}

(c) The Authors 2020. This is an Open Access article, distributed under the terms of the Creative Commons Attribution-

NonCommercial-NoDerivatives licence (http://creativecomm org/licenses/by-nd/4.0/), whic permits non-commercial re-use distribution, and reproduction in any medium, provided the original work is unaltered and is properly cited. The written permission of Cambridge Universty Prission of Cambridge University Press must be obtained for com mercial re-use or in order to create a derivative work.
France has a long tradition of concern for maternal and perinatal mental health. However, the national organisation of psychiatric care does not yet provide structured guidelines on the organisation of perinatal psychiatric care. This paper provides an update on existing resources and their linkage to primary care and obstetric and paediatric services, as well as a review of current and future national priorities that are under development.

France's tradition of concern about maternal perinatal mental health began with the French psychiatrists Esquirol $^{1}$ and Marcé, ${ }^{2}$ who first described maternal psychopathology emerging during the perinatal period. Their reports of the existence of specific maternal psychopathological disorders in the perinatal period should be considered in the light of changes that were occurring in French society at that time. Successive revolutionary assemblies had initially granted women some rights, including the right to divorce and to inherit on an equal footing with men. On the other hand, the violence sometimes suffered by children was also becoming recognised at this time, especially through the works of A. Tardieu, professor of forensic medicine in Paris, who first described the 'beaten child syndrome'.

In 1945, following the Second World War, a nationwide community-based mother and child protection service was established. This initiative, Protection Maternelle et Infantile (P.M.I.) was to be free of charge. It was designed to support families from pregnancy until the child's third birthday. This service provided preventive care, by means of a follow-up service for children from before birth, with regular developmental screenings and vaccinations following national guidelines. P.M.I. also provides supportive home visits by midwives and nursery nurses during the perinatal period, taking into account maternal mental health. Thereafter, the first mother-baby hospital admissions were carried out in the early $1960 \mathrm{~s},{ }^{4}$ while a group of French child psychiatrists, including Myriam David and Serge Lebovici, founded the discipline of infant psychiatry.

Other adult psychiatrists such as Thérèse Lempérière $^{5}$ have continued to build upon Marcé's work on perinatal parental psychiatric disorders. Reflecting the fact that for many years there has been a bipartite evolutionary dynamic of adult and infant psychiatry in France, the first full-time mother and baby unit (MBU), opened in Créteil in 1979, was in a child psychiatry department, whereas the second unit, which opened in 1980, was in an adult psychiatry department at the Paul-Brousse Hospital in Villejuif.

\section{Current general organisation of perinatal mental health services}

The organisation of French health services is centralised at a national level under the control of the Ministry of Health. Within this ministry, the General Health Directorate (Direction Générale de la Santé) prepares public health policy and contributes to its implementation. In addition to this work on the main areas of public health, the General Directorate of Healthcare Supply (Direction Générale de l'Offre de Soins) is in charge of the organisation of the care provided by the various health services. Regional structures (Agencies Régionales d'Hospitalisation) regulate and control the health budget at a regional level, in accordance with priorities defined at a national level. In addition to these ministerial structures, the High Authority of Health (Haute Autorité de Santé), an independent scientific public authority that aims to ensure quality in the health, social and medico-social fields for the benefit of individuals, regularly provides recommendations for good practice. 